\title{
VI.
}

(Aus der medicinischen Klinik in Heidelberg.)

\section{Ein Fall von congenitaler halbseitiger Gesichtshypertrophie.}

\author{
Yon Dr. Franz Ziehl, Assistenzarzt an der Klinik.
}

Vor einiger Zeit gelangte auf der Heidelberger medicinischen Klinik ein Fall von halbseitiger Hypertrophie des Gesichtes zur Beohachtung, der allein schon durch die Seltenbeit seines Vorkommens einiges Interesse erregen diurfte.

P. S., 4jäbriger Sohn eines Bauern aus Z., stammt aus ganz gesunder Familie, Weder in der des Vaters noch in der der Mutter sollen jemals Störungen in der Entwicklung des Körpers oder Nervenkrankheiten vorgekommen sein. Auch die beiden Gesehwister desselben, ein Bruder von 12 Jahren und eine Schwester von 10 Jahren, sind ganz gesund und körperlich wohlgebildet.

Die Mutter giebt an, dass der Knabe zur rechten Zeit geboren wurde. Die Zeit der Schwangerschaft und die Geburt verliefen obne besondere Zwischenfälle. Jedoch will die Mutter einmal, etwa 4 bis 5 Wochen vor der Niederkunt einen grossen Schreck erlitten baben, indem ihr ein Haufe Brennessel auf die rechte Seite des Gesichtes fiel. Dadurch soll nach ihrer Meinung die an gleicher Stelle sich findende Difformität im Gesicht ihres Kindes zu Stande gekommen sein.

Gleich nach der Gebart des Kindes wurde bemerkt, dass die rechte untere Gesichtshälfte desselben voluminőser war als die liake. Doch wurde die darüber sehr erschrockene Mutter damit getröstet, dass dies nur eine durch den Drack wăbrend der Geburt entstandene Geschwulst sei, dle sich binnen wenigen Tagen wieder verlieren würde. Dies trat jedoch nicht ein, vielmehr wurde nach einigen Tagen entdectst, dass dasselbe Missverbältniss in der Entwicklong zwischen rechts und links vicht nur an den Weichtheilen des Gesichtes, sondern auch an den Gebilden in der Muddhöhle vorhanden war. Auch mit dem Wachsthum des Kindes hat darin eine Ausgleichung nicht stattgefunden, vielmehr ist das Verhăltniss zwischen rechts und links etwa immer das gleiche geblieben; nur die rechte Tonsille hat auch extrauterin eine langsam fortschreitende Hyperplasie gezeigt und in der letzten Zeit durch ihre Grôssenzunahme bedrohliche Erscheinungen bervorgerufen.

Der Knabe wurde awei Jahre hindurch von der Mutter gestillt, soll immer gut genăhrt ausgeseben baben, lernte aber das Sitzen erst in einem Alter von anderthalb Jahren, Stehen und Laûen erst im letzten halben Jahr. Die ersten Zäbne bekam er, als er fünf bis sechs Monate alt war. Ein Unterschied in dem Hervortreten derselben auf der rechten hypertrophischen oder der linken normalen Seite 
wurde nicht bemerkt. Die Zăhne wurden aber bald schlecht, brachen ab oder fielen wieder aus.

Als der Knabe fünf Wochen alt war, traten zum ersten Mal Krämpfe bei ihm auf, dte die gesammte Körpermusculatur ergriffen. Seitdem wiederholen sich dleselben oft drei bis vier Mal in der Woche, oft setzen sie fünf bis sechs Wochen lang ganz aus. Das Wiedereintreten derselben will die Mutter gewöbnlich dadurch vorausseben können, dass der sonst normale Urin des Knaben an Menge abnimmt, dunkler und nach geringem Stehen wolkig und trübe wird. Die Krämpfe dauerten zuweilen aur einige Minuten, zuweilen länger, einmal sogar zwei Stunden hindurch so heftig, dass man für das Leben des Knaben fürchtete. Wenn dieselben etwas länger dauern, pflegt dabei ein so starkes Schwitzen einzutreten, dass das ganze Bett durchnässt wird. Während die Krämpfe anfangs belderseits gleich stark waren, sollen sie später auf der linken Selte oft viel heftiger gewesen sein als auf der rechten, die zuweilen selbst ganz unbetheiligt blieb. Die Augen soll der Knabe dabei stets nacb der linken Seite hin verdreben. Ob auch an dem Schwitzen vielleicht die eine Seite des Körpers mehr betheiligt ist als die andere, kann die Mutter nicht angeben.

Von den ersten Monaten abgesehen, bat der Knabe Urin und Koth nie unter sich gehen lassen, sondern stets durch Unruhe etc. sein Bedürfniss ansgedrückt.

Sprechen hat derselbe bisher nicht gelernt, wenige ziemlich unverstăndliche Worte ausgenommen, doch rührt dies vielleicht von der abnormen Beschaffenheit der Zunge her; dafür sucht sich das Kind durch allerlei Zeichen mit den Händen verstăndlich zu machen. Die geistige Entwicklung desselben bat in letzter Zeit erheblich zugenommen. Es spielt und ist ziemlich lebhaft geworden, während es die ersten drei Jahre hindurch sehr apathisch gewesen seln soll.

In dea letzten Monaten batte die rechte Tonsille eine solche Grösse durch fortschreitendes Wachsthum erreicht, dass das Kind im Schlafe oft laut schnarcht und beim Liegen auf der linken Seite dyspnoisch wird. Dies war die Veranlassung, wesbalb die Eltern das Kind auf die hiesige Klinik brachten.

Gleich beim ersten Anblick des Knaben fällt eine erhebliche Differenz in dem Volum der beiderseitigen Gesichtshälften anf und zwar zn Gunsten der rechten Seite. Diese Differenz betriff jedoch nur den Theil des Gesichtes, der unterbalb einer durch die Augen und die Nasenwurzel gezogenen Querlinie liegt. Dieser ist rechts um vieles umfangreicher als der entsprechende Theil links. Die Volumszunabme erstreckt sich auch etwas auf den Hals nach abwärts. Bel weitem am meisten sind daran die rechte Wange und die rechte Hälfte des Kinns betheiligt. Die Nase steht nicht genan medial, sondern ist etwas nach links verschoben, wohl in Folge der Hypertrophie der rechten Seite, im Uebrigen aber ist sie wohlgebildet und zeigt keine Differenzen in der Grösse der beiden Hälften, auch die Dicke der Nasenflügel ist beiderseits die gleiche. Das Debmliche Verhalten zeigt der Mund: er ist gleichfalls etwas nach links verschoben, Differenzen in der Grösse oder der Dicke der beiderseitigen Líppenhälften lassen sich weder für die oberen noch für die unteren Lippen erkennen. Der Mund wird beständig etwas geöffnet gehalten, und fortwährend fliesst Speichel aus demselben heraus.

An den oberhalb der erwähnten Linie gelegenen Theilen finden sich ketne 
Differenzen beiderseits. Dỉe beiden Stirn- und Schỷdelbäliten sind genau symmetrisch. Die Nähte am Schädel sind überali geschlossen, ełenso die Fontanelle. Dagegen ist der Schädel im ganzen genommen grősser als dem Alter des Knaben entsprechen würde. Der Umfang des Kopfes beträgt $55 \mathrm{~cm}$, übeririff also das Durchschnittsmaass für den Kopfumfang eines vierjäbrigen Knaben um $5 \mathrm{~cm}$, das Maximum des Comlangs für dasselbe Alter noch um $2 \mathrm{~cm}^{\mathbf{1}}$ ).

Die Haare stehen auf beiden Kopfhälften gleich dicht und sind von gleicher Farbe, jedoch sind die Harre binter dem rechten Obr nicht so weich anzutulhlen wie links; sie erscheinen struppiger, rauher und trockner für das Gefühl. Haarwuchs an abnormen Stellen im Gesicht findet sich nicht.

An den Augenbranen, an den Augenlidern, den Cillen, den Augenspalten besteht tein Unterschied zwischen belden Seiten. Auch die Bulbi sind von gleicher Grösse. Es zelgt sich ein mässiger Strabismus convergens des rechten Auges, das zugleich ein wenig mehr nach rorn steht als das linke, Auch ist der Durchmesser der rechten Iris grösser als der der linken. Auffallende Differenzen in der Färbung der rechten nod linken Iris sind nicht zu erkennen. Die Pupillen sind gleich und reagiren beiderseits glelch gut auf Licht. Die Untersuchung mit dem 0phthalmoskop zeigt, dass reohts eine ziemlich starke Myopie besteht, links nicht.

Den Kopf hält das Kind nicht ganz grade, sondern senkt tho beständig etwas auf die linke Schulter (vielleicht um denselben wegen der grösseren Schwere der rechten Seite so besser balavzilen za können ?).

Sehr merkwürdig ist das Verhalten der rechten Obrmuschel. Sie ist viel dicker, länger and breiter als die linke, hesonders ist anch das Obrläppchen viel voluminöser. Die Höhe der 0hrnuschel beträgt liaks $5,5 \mathrm{~cm}$, rechts dagegen $8 \mathrm{~cm}$; die Breite rom hinteren, äusseren Rande derselben bis zur Spitze des Tragus links 2,5 , rechts $3,5 \mathrm{~cm}$. Auch der assere Gehörgang ist rechts etwas weiter als links. Soweit eine solche Prüfung bei dem Knaben überhaupt möglich war, ist das Gehörsvermögen auf beiden Seiten gleich gut.

An verschiedenen Stellen dẹs Gesichtes finden sich Pigmentanbäufungen von brauner Farbe. An der rechten. Stirnhälfte sieht man einen solchen schmalen Pigmentstreifen von oben nach unten verlaufen, die ganze rechte Ohrmuschel ist bräunlich gefärbt, am stärksten pigmentirt ist jedoch die Gegend längs des rechten Unterkieferrandes ond die vordere Hälfte der rechten Halsseite. An letzterer Stelle ist die Farbe am dunkelsten, die Haut raub, verdickt und durch Langs- und Querfurchen wie in einzelne kleine Felder getheilt. Die Pigmentirung setat sich noch eine Strecke weit af die Brust fort, ühersehreitet aher die Mittellinie nicht.

An den knöchernen Theilen lassen sich bei der äusseren Untersuchung derselben (Augenhöble, Ober- and Unterkiefer, Warzenfortsatz, Jochbein) keine Differenzen zwischen beiden Seiten erltennen; es scheinen daher nur die Weichtheile an der Hypertrophie betheiligt zo sein.

Sehr merkwürdig ist die Theilaahme der Gebilde innerhalb der Mundhöhle an der Hypertrophie. Hier scheinen jedoch auch die knöchernen Theile betheiligt zu

) Nach Bagiasky, Rachitis. 
sein, wenigstens insofern als die Alveolarfortsätze des Ober- und Unterkiefers rechts breiter siṇd als links. Diese Differenz ist am hinteren Ende am grössten und nimmt von da nach vorn hin allmählich ab. Im Uebrigen sind jedoch die Kíeferknochen an beiden Selten gleich entwickelt. An den Zähnen bemerkt man keinen Unterschied zwischen rechts und links. Sie sind übrigens theilweise ausgefallen und die noch vorhandenen sehr schlecht und cariös.

Auch die rechte Tonsille ist ganz erheblich vergrössert und mindestens von der Grösse einer Wallnuss. Auch die Theilnahme der rechten Tonsille an der Hypertrophie wurde, wie schon erwähnt, bald nach der Geburt bemerkt. Die ubrigen Theile des weichen Gaumens und des Rachens zeigen rechts und llaks keine Differenzen.

Am sonderbarsten ist das Verhalten der Zunge. Dieselbe ist in ibrer rechten Hälfte bedeutend, um das zwei- bis dreifache, hypertrophisch, so dass die linke Hälfte derselben wie verdrängt und nur als ein Anhängsel der rechten erscheint und die abgestumpfte Zungenspitze ganz und gar von der rechten Hälfte gebildet wird. Ausserdem ist die Zunge in Folge der Hypertrophle der rechten Hälfte im Verbältniss zur Mundböhle etwas zu gross und steht daher mit der Spitze etwas zum Munde heraus. Die Papillae fungiformes der rechten Hälfte sind enorm vergrössert und treten als kleine Knöpfe von $2 \mathrm{~mm}$ Höbe und 1-2 mm Durchmesser hervor, so dass die Zunge eine himbeerartige Oberfläche zeigt. Desgleichen sind auch die Pap. filiformes auf der rechten Seite länger und dicker als links. Die Pap. circumvallatae verbalten sich auf beiden Seiten gleich.

An den Lymphdrüsen des Halses und der Unterkiefergegend lässt sich nichts Abnormes finden.

Es besteht eine ganz erheblich vermehrte Secretion des Speichels, der beständig aus dem Munde herausfliesst. Wie es scheint geht dieselbe von den Drüsen der rechten Seite aus. Doch lässt sich das mit Sicherheit nicht feststellen, eine Vergrösserung der Speicheldrüsen der rechten Seite ist jedoch durch die Palpation nicht zu ermitteln.

Geschmack, Geruch und die Sensibilitäł konnten wegen des jugendlichen Alters des Kaaben nicht geprüft werden.

Die Bewegungen der Gesichtsmuskeln gehen beiderseits gleich gut von statten.

Unterschiede im Palse der Carotiden, der Temporal- und Maxillararterien der belden Seiten sind nicht nachzuweisen. Eine vergleichend thermometrische Messung in beiden Gehörgängen giebt kein sicheres Resultat wegen der Unruhe des Kindes.

Die Ernährungsverhăltnisse des Körpers sind aussergewöhnlich gut. Das Körpergewicht des Kindes beträgt $20,5 \mathrm{~kg}$, seine Grösse $93 \mathrm{~cm}$. Besonders das Unterhautfettgewebe ist am ganzen Körper sehr stark entwickelt.

Am Rumpf und an den Extremitäten lassen sich Unterschiede zwischen rechts und links nicht erkennen. Vielleicht ist die rechte Hand etwas grösser als die linke, aber wohl nur deshalb, weil das Kind dieselbe von je her mehr gebraucht hat als die linke.

Díe Untersuchung der Brust- und Unterleibsorgane erglebt nichts Bemerkenswerthes. 
Nach dem ausfuhrlich mitgetheilten Status handelt es sich bei dem Knaben um eine halbseitige Hypertrophie des Gesichtes und der angrenzenden Theile der Mundhöhle und zwar ist sie, wie die Anamnese ergiebt, angeboren. Im extrauterinen Leben scbeint dieselbe im Allgemeinen nicht mehr, jedenfalls nicht erheblich progressiv gewesen zu sein, nur an der rechten Tonsille zeigt sich eine beständige Zunahme in der Grösse, die dem übrigen Wachsthum des Kindes nicht entspricht. Es ist also bier der hypertrophisebe Prozess noch nicht zum Stillstand gekommen. Ausserdem weist der beständige Speichelfluss auf abnorme Reizungszustände in den die Speichelsecretion beherschenden Nerven hin.

Derartige Fälle von halbseitiger Gesichtsbypertrophie scheinen ausserordentlich selten zu sein. Soweit wir sehen, finden sich davon nur vier Fälle in der Literatur erwähnt.

Der erste wurde von Friedreich ${ }^{\mathrm{a}}$ ) beschrieben. Bei dem zur Zeit der Beobachtung sechzehn Jahre alten Mädchen betraf die Hypertrophie die rechte Gesichtshälfte, wurde gleich bei der Geburt bemerkt und trat im Verlauf der Jahre noch markirter hervor. Die Aehnlichkeit zwischen dem Friedreich'schen Fall und dem unsrigen ist so gross, dass die dort gegebene Beschreibung fast wörtich auf den unsrigen passt. Ein Hydrocephalus war jedoch nicht vorhanden, und das Mädchen zeigte ausser der Hypertrophie des Gesichtes keine weitere Anomalie weder in psychischer noch in pbysischer Beziehung. Sie soll im Gegentheil geistig so entwickelt gewesen sein, dass sie in der Schule immer unter den Ersten sass.

Die Hypertrophie betraf die Weichtheile des Gesichtes, das Ohr, die Tonsille und die Zunge. Die Knochen waren nicht mit ergriffen, ausgenommen die Alveolarfortsătze. Es fanden sich abnorme Pigmentirungen in der Haut und Haarwuchs an ungewöhnlichen Stellen. Auch die Talgdrüsen auf der rechten Obrmuschel und dem rechten Zitzenfortsatz waren hypertrophisch, ragten als weisse, bis selbst hirsekorngrosse Knötchen herror, und diese Gegend war - wohl in Folge einer Hypersecretion - von einer mächtigen, stellenweise continuirlichen, schmierigen Talgschicht überzogen. Die Schweisssecretion und die Schweissdrüsen waren beiderseits gleich, dagegen bestand eine reichliche Speichelabsonderung

3) Dieses Archiv. 1863. Bd. XXVIII. S. 474. 
auf der rechten Seite. Trotz der Difformität der Zunge war an der Sprache nichts Auffälliges zu bemerken. In den Functionen des Hörens und Riechens bestanden keine Unterschiede beiderseits; dagegen war die Geschmacksperception und die Sensibilität auf der hypertrophischen Zungenhälfte vermindert, auf der Haut war dagegen letztere beiderseits gleich. Die Gesichtsmuskeln functionirten gut. Temperaturunterschiede auf beiden Seiten konnten nicht sicher gefunden werden, obgleich das Mädchen mit Entschiedenheit oftmals ein Gefühl stärkerer Wärme an der rechten Wange zu haben angab und auch die Haut letzterer durchschnittlich von dunklerer Röthung erschien. Das Mädchen litt häufig an rechtsseitigen Zahnschmerzen.

Einige Zeit nach der Beobachtung starb dasselbe an einem Ileotyphus. Bei der Section wurde nichts gefunden, was zu einer Erklärung hätte herangezogen werden können. Ausser dem schon im Leben beobachteten fand sich nur, dass auch das rechte Felsenbein hypertrophisch, dicker, prominenter und an der Oberfläche mit gröberen Höckern versehen war als das linke. Zwischen den beiden Gehirnhälften fand sich keine Differenz, ausgenommen eine leichte Vertiefung der Gehirnbasis an der dem hypertrophischen rechten Felsenbein entsprechenden Seite; auch die von der Schädelbasis abtretenden Nervenstämme waren nicht verändert.

Ein zweiter Fall ist von Heumann beobachtet worden und im Anschluss an den vorigen von Friedreich beschrieben. Zur Zeit der Beobachtung war der Knabe 5 Jahre alt. Auch hier bestand die Difformität seit der Geburt. Die linke Backe, der Ober- und Unterkiefer nebst den Zähnen der linken Seite, die linke Hälfte der Zunge, und auf dieser besonders hervortretend die Papillen, waren fast doppelt so gross wie rechts. Die linke Ohrmuschel war zwar nicht auffallend grösser, aber die Haut derselben bedeutend dicker anzufühlen als rechts. Die Haare der linken Seite waren stärker und vor dem $0 \mathrm{hr}$ tiefer herabgewachsen als auf der anderen Seite. Seit der Geburt litt das Kind beständig an einem bellgelben Ohrenfluss auf der bypertrophischen Seite bei ungestörtem Gehör, sowie an einem lästigen Speichelfluss aus dem linken Mundwinkel.

Ein dritter Fall ist von Passauer ${ }^{1}$ ) beschrieben. Auch bei

7) Dieses Archiv. 1866. Bd. XXXVII. S. 410.

Arohiv f. pathol. Anat. Bd. XCr. Hft. 1. 
diesem, zur Zeit der Beobactung 11 Jahre alten Knaben war die Hypertrophie der linken Gesichtsseite solort bei der Geburt bemerkt worden und hatte sich mit dem Wachsthum des Knaben gleichmässig fortgebildet. Derselbe war geistig ganz normal entwickelt, sogar besser als seine Geschwister, wurde von seinem Lehrer wegen seiner Fãhigkeiten und Fortschritte gelobl und las fliessend und verständig. Die Hypertrophie betraf die linke Gesichtshälfite besonders die Wange, die linke Hallte der Zunge mit besonders auffallender Betheiligung der Papillen, die Zähne, die Zahnbögen und das Zahnfleisch, das Gaumensegel war normal. Die linke Wange war ausserdem stärker gerōthet als die rechte. Ein Mienenspiel bestand im Bereiche der Hypertrophie nicht. ob die Knochen betheiligt waren, ist aus der Beschreibung nicht zu entnehmen; es findet sich nur die Bemerkung, dass der untere Rand des Unter-kiefers rechts von der Protub. ment. bis zum Ang. maxill. $1,5 \mathrm{~cm}$ wenigey maass als links, ob jedoch dieser Unterschied durch eine verschiedene Grösse des Unterkiefers oder nur durch die dickeren Weichtheile bedingt war, ist nicht angegeben.

Eine vierte Beobachung findet sich endlich kurz bei Beck ${ }^{1}$ ) erwähnt. Es handelte sich um ein dreijäbriges vollkommen gesundes und Kräftig gebautes Mädchen. Gleich bei der Geburt desselben wurde eine grössere Fülle der rechten Wange beobachtet. Mit dem Wachsthum des Kindes hielt diese gleichen Schritt. Die Wange hing in Form einer derben Falte bis zum Rande des Unterkiefers herab und zog den Mundwinkel nach abwirts. Die Falte wurde nur durch Hant und Zellgewebe gebildet. Naevi fanden sich nicht, vielmehr bestand der Zustand ohne Entarkung der Gebilde und ohne ein fremdartiges Bildungsverhältniss.

In allen diesen früheren Fällen handelt es sich also um Kinder, die ausser der halbseitigen Hypertrophie des Gesichtes weder geistige noch körperliche Anomalien darboten. Wir dürfen daraus für unseren Fall schliessen, dass der hier sich findende Hydrocephalus denn darauf deuten der grosse Umfang des Kopfes und die Anfälle von Convulsionen hin - nur eine Complication ist, die mit der Gesichtshypertrophie nicht unmittelbar in Zusammenhang steht.

In allen bisher beschriebenen Fällen wurde die Hypertrophie gleich bei den Geburt benerkt und hielt mit dem übrigen Wachs-

1) Med. Anualen von Pachelt, Chelius und Nägele. 1836. S. 89. 
thum des Kindes entweder gleichen Schritt oder war sogar noch etwas progressiv [Fall Friedreich, bei unserem Fall die Tousille $\left.{ }^{1}\right)$ ]. Nur ein Mal war die Wange allein befallen (Fall Beck), in allen übrigen erstreckte sich die Hypertrophie auch auf die benachbarten Theile: Ohrmuschel, Zunge, Gaumen, Tonsille, Zähne, ein Mal auch auf den Ober- und Unterkiefer (Fall Heumann); öfters fanden sich abnorme Pigmentanhäufungen in der Haut oder abnormer Haarwuchs. Besonders hervorgehoben zu werden verdient, dass sich oft neben den abnormen anatomischen Verhältnissen auch abnorm functionelle fanden: anhaltender Speichelfluss (Fall Friedreich, Heumann, unser Fall), Secretion aus dem ohr (Fall Heumann), übermässige Talgdrüsensecretion (Fall Friedreich), öftere stärkere Röthe der Wange (Fall Friedreich, Fall Passauer), Lähmung der Musculatur im Bereich der Hypertrophie (Fall Passauer), abnormes Wärmegefühl daselbst (Fall Friedreich), häufige Anfälle von Zahnschmerzen (Fall Friedreich).

Die Uebereinstimmung dieser Fälle unter einander ist so gross, dass wohl der Schluss erlaubt ist, es sei anch die Pathogenese für alle die gleiche oder mindestens eine sehr ähnliche.

Die halbseitige Hypertrophie war bisher zwei Mal der Gegenstand monographischer Darstellung, zum ersten Mal durch Trélat und Monod ${ }^{2}$ ), das zweite Mal durch Lannelongue ${ }^{3}$ ). Beide stellen die bisher beobachteten Fälle von halbseitiger Hypertrophie des Gesichtes in eine Gruppe zusammen mit der viel häufiger beobachteten, wo eine obere oder eine untere Extremität, resp. beide zugleich hypertrophisch waren, gehen aber in ihren Ansichten über die Entstehung derselben weit auseinander.

Trélat und Monod glauben die Hypertrophie durch vasomotorisch-trophische Einflüsse erklären zu mülssen, und zwar soll es sich um eine unvollständige Lähmung der vasomotorisch-trophischen Nerven handeln, die sich auf eine Hälfte oder auf einen Theil einer Hälfte des vasomotorisch-trophischen Nervensystems beschränkt.

1) Auch das rechte $0 \mathrm{hr}$ zeigt eine so abnorme Grösse, dass es nicht unwahrscheinlich ist, auch hier sel die Hypertrophle extrauterin noch progressiv gewesen, resp. sei es noch.

2) De l'hypertrophie unilatéral partielle ou totale da corps. Archives générales de médecine. 1869. p. 536.

3) De l'hypertrophle unilatérale. Thèse. Paris 1874. 
Dadurch soll die Blutcirculation verlangsamt werden, und die langsamere Circulation des Blutes soll die Hypertrophie zu Stande bringen. Zur Stütze dieser Ansicht wird auf Experimente von Cl. Bernard und Schiff verwiesen, wo nach Durchschneidung der Vasomotoren schliesslich eine Hypertrophie im Bereich der gelähmten Gefässe eintrat. Uebrigens verhehlen Trélat und Monod sich keineswegs, dass, wenn so auch die Hypertrophie zu erklären sei, doch damit immer noch dunkel bleibt, weshalb die Lähmung der Vasomotoren eingetreten sei, resp. weshalb sie auf die eine Seite besebränkt blieh.

Diese Theorie wird von Lannelongue gänzlich verworfen, weil die einzelnen Theile des Embryo sich unabhängig von einander entwickelten, weil der Einfluss des Nervensystems auf die Ernährung dazu selbst bei der Geburt noch viel zu gering sei und weil ollier bei einer Wiederholung der Schiff'schen Experimente nach Durchschneidung der Vasomotoren trotz der Gegenwart einer grösseren Menge von Blut in den gelähmten Theilen doch keine Hypertrophie derselben eintreten sah. Nach seiner Ansicht soll ein Reiz noch unbekannter Art auf die Epiphysenknorpel und das Periost stattgefunden haben, dadurch soll eine Hypertrophie der Knochen za Stande kommen und erst secundär sich eine Hypertrophie der Muskeln u. s. w. anschliessen.

Zugegeben selbst, dass diese Ansicht für die halbseitige Hypertrophie der Extremitäten richtig sein könnte, so kann sie doch unmöglich zu einer Erklärung der halbseitigen Gesichtshypertrophie dienen, da grade hier in den meisten Fällen die Knochen an der Hypertrophie nicht betheiligt waren. Es ist daher geradezu unbegreiflich, wie Lannelongue die hier in Rede stehenden Fälle in seine Casuistik aufnehmen konnte oder wie er Angesichts derselben uberhaupt zu seiner Theorie gekommen ist. Dem gegenüber bat die Trélat-Monod'sehe Ansicht wenigstens den Vorzug, logisch möglich zu sein.

Sieht man sie als richtig an, so bieten die hier besprochenen Fälle eine interessante Parallele dar zu der halbseitigen Gesichtsatrophie, die ja jetzt meist auf vasomotorisch-trophische Störungen zurückgefuhrt wird. Dass in allen bisher beobachteten Fällen die Hypertrophie intrauterin entstand, wahrend die halbseitige Atrophie erst im extraterinen Leben beginnt, kann diesen Vergleich um so 
weniger verbieten, als von Emminghaus ${ }^{1}$ ) auch ein Fall von angeborner halbseitiger Atrophie beschrieben worden ist.

Bei dem zur Zeit der Beobachtung 12 Jahre alten Mädchen war die linke Stirn abgeflacht, die linke Lidspalte enger, die linke Pupille excentrisch gelegen, der ganze Bulbus etwas atrophisch, die Thränenabsonderung links stärker. Die Nase stand nach der linken Seite, der Oberkiefer war eingesunken, der Unterkiefer stark verkleinert. Die Weichtheile des Mundes links viel kleiner, namentlich deutlich an der Zunge, unregelmässige Zahnstellung. Im Gesicht konnten keine circumscripten Hautatrophien gefunden werden, auch war die Behaarung gleich. Die Bewegungen der Gesichtszüge boten keine anderen Asymmetrien als die durch die Kleinheit der linken Seite erklärlichen. Der Zustand wurde schon bei der Geburt constatirt. Auch die linke obere Extremität war $1 \mathrm{~cm}$ kürzer als die rechte.

Emminghaus meint zwar, der Umstand, dass die Anomalie gleich bei der Geburt wahrgenommen wurde und dass er keine Fortschritte der Volumsabnahme aufwies, stelle diesen Fall mit den gewöhnlichen Fällen nicht auf gleicte Stufe, es handle sich hier um ein Kleinergebliebensein, nicht um ein Kleinergewordensein der Theile, wie es in der wahren Gesichtsatrophie resultire; aber, wenn auch zuzugeben ist, dass die Genese der Atrophie in beiden Fällen eine verschiedene sein mag, so kann nichtsdestoweniger, wie uns scheint, wenn man sich auf den Boden der neurotischen Theorie stellt, die Aetiologie (der Fortfall des trophischen Reizes) doch die gleiche sein. In dem einen Fall (späterer, extrauteriner Beginn) werden die Theile nach Fortfall des trophischen Reizes zu atrophiren beginnen, im anderen Fall (früher intrauteriner Beginn) zu wachsen aufhören und in dem jeweiligen Zustand verharren. Das Resultat muss in beiden Fällen das gleiche sein. Die Fälle sind daher trotz verschiedener Genese doch als zusammengehörige zu betrachten und bilden nur eine Gruppe. Es kann uns daher auch der meist extrauterine Beginn der Hemiatrophie in dem Vergleich mit der Hemihypertropbie nicht hinderlich sein.

Auch der Umstand, dass bei der Hemiatrophie das Leiden längere Zeit progressiv ist, steht dem nicht entgegen, denn erstens

1) Ueber halbseitige Gesichtsatrophie. Deutsches Archiv für kllinlache Medicin. 1873. Bd. XI. S. 96. 
muss es ausserordentlich schwer sein, eiren etwaigen fortschritt der Hypertrophie sicher zu constatiren, wejl die Kinder noch im Wachsthum begriffen waren und daher ein sicherer Maassstab zum Vergleich an der gesunden Seite fehlt, man vielmehr auf ein Schäłzen des relativen Verhältnisses beider Gesichtshälften zu einander angewiesen ist, so dass es keineswegs sicher ist, ob nicht etwa doch in allen Fallen nach der Geburt noch eine zeitweilige Zunabme der Hypertrophie stat hatte. Zweitens wurde trotz dieser entgegenstehenden Schwierigkeit wenigstens in dem Friedreich'schen Fall eine Zunahme der Hypertrophie während der Entwicklung constatirt. Drittens ist auch die Atrophie nicht in infinitum progressiv, sondern kommt nach einiger Zeit [nach Wette ${ }^{x}$ im Allgemeinen nach 2-4 Jahren] zum Stillstand. Also ist anch von dieser Seite aus ein Vergleich der Hemiatrophie mit der Hemibypertrophie wohl erlaubt.

Die bisher beschriebenen 43 Fälle von halbseitiger Gesichtsatrophie sind von Wette (l. c.) in vier Gruppen gebracht worden nach der Ausdehnung der Atrophie:

Gruppe AI. Totale halbseitige Gesichtsatrophie aller Theile. Es sind betroffen Haut, Panniculus adiposus, Muskeln, Knochen des Gesichtes, Nase, Ohr, Gebilde der Mundhöhle und Haare, Exophthalmus. - Zuweilen excl. des einen oder anderen der aufgezählten Theile.

Gruppe A II. Totale halbseitige Gesichtsatrophie nur der Weichtheile. Es sind betroffen Haut, Fett, Muskeln und Haare. Ein Mal incl. Zungenhälfte, einige Mal excl. Muskeln.

Gruppe BI. Partielle balbseitige Gesichtsatrophie (Knochen und Weichtheile). Es sind betroffen einzelne Knochen, die darüber liegende Haut, Panniculus adiposus, Haare.

Gruppe B II. Partielle halbseitige Gesichtsatrophie (Haut und Unterhautzellgewebe).

Ausserdem fanden sich in unregelmässiger Vertheilung auf die verschiedenen Gruppen: abnorme Pigmentirungen bald von weisser (? Mangel des Pigments), bald gelber bis brauner, ein Mal violetter Färbung, einige Mal hatte die betroffene Gesichtshälfte eine auffallend blasse Farbe, zwei Mal war die Motilität der Muskeln ganz

1) Annalea der städtischen allgemeinen Krankenhäuser zu München. 1876 und 18\%. $\$ .600$. 
aufgeboben. Die Sensibilität zeigte sich in fast allen Fällen an den atrophischen Partien erhöht (was nach Wette wohl seine Erklärung in der dünueren Haut und dem Fehlen des Panniculus adiposus findet; dem entgegengesetzt erklärt Friedreich die in seinem Fall beobachtete Herabsetzung der Geschmacksperception und der Sensibilität an der Zunge aus einer wegen der Hypertrophie der Zungenpapillen bestehenden grösseren Entfernung der Nervenendigungen von der freien Zungenoberfläche). Die Secretion der Talgdrusen scheint in den meisten Fällen herabgesetzt gewesen zu sein, die Schweisssecretion war einige Mal aufgehoben oder berabgesetzt, ein Mal war die Speichelsecretion erheblich vermehrt, Temperaturerniedrigung fand sich vier Mal, oft bestanden neuralgische Zabnoder Kopfschmerzen.

Wie man leicht sieht, zeigen die Fälle von halbseitiger Hypertrophie von Allem grade das Gegentheil. Das Gegenstuick der Gruppe AI ist der Fall Heumann; von A II der Fall Friedreich, Passauer, unser Fall; von B II der Fall Beck. Ebenso sind die functionellen Symptome öfters einander ganz entgegengesetzt, zuweilen allerdings auch die gleichen.

Es ergiebt sich daraus ganz von selbst die Frage, ob nicht vielleicbt auch die Aetiologie in den beiden Reihen von Fällen einander entgegengesetzt sein könne.

Für die halbseitige Gesichtsatrophie wird wohl von den meisten jetzt ein neurotischer Ursprung als sicher angenommen. Beruht nun auch die halbseitige Gesichtshypertrophie auf einen solchen?

Wie schon oben erwähnt ist, suchen Trélat und Monod in der That die in Rede stehenden Fälle durch einen Einfluss von Seiten des Nervensystems zu erklären und zwar durch eine vasomotorische Paralyse.

Wie bereits erwähnt, haben Cl. Bernard und Schiff nach Durchschneidung von vasomotorischen Nerven Hypertrophie entsteben seben, was allerdings von Ollier wieder bestritten wurde. Später hat dann Mantegazza') die Sache einer erneuten Untersuchung unterworfen und die histologischen Veränderungen studirt, die nach Durchschneidung des Nerven in den Geweben anftreten. Er fand, dass nicht nur Atrophien, sondern auch Hypertrophien die Folge

1) Giornale Venet. di scienze mediche ser. 3. tom, 6. 1867. Nach VirchowHirsch, Jahresbericht $186 \%$. 
von Nervenverletzungen sein können, z. B. beobachtete er Hypertrophie des Bindegewebes und Periostes, Hypertrophie der Muskelsubstanz, Osteophytbildung etc.

Vielleicht gehört dahin auch eine Beobachtung von Stilling ${ }^{1}$ ), wo in Folge eines Steinwarfes gegen das Gesicht im 9. Lebeusjahr eine halbseitige Hypertrophie desselben eintrat. Es handelte sich um eine 38jäbrige Frau, bei der die rechte Gesichtshälfte bedeutend vergrössert war. Die Haut war von normalem Aussehen und Colorit, die Geschwulst weich, von Mannsfaustgrösse und mit einer teleangiectatischen Erweiterung der kleineren Haut- und Schleimhautgefässe verbunden. Die Mundschleimheut erschien im rechten Mundwinkel hervorquellend, mit vielen erweiterten rothen Blutgefässen versehen, die Wangenschleimbaut dieser Seite bei geöffnetem Munde bläulichroth, durkel aufgelockert, ebenfalls mit unzäbligen erweiterten Blutgefässen durchsetzt. Die grösseren Arterien des Gesichts zeigten keine Erweiterung. Sensibilităt und Motilität der befallenen Gesichtshälfte waren vollkommen intact, die Bewegungen gingen so gut wie auf der linken Seite von Statten. Stilling nimmt an, dass der Wurf den $N$. infraorbitalis verletzt habe und dadurch eine völlige Paralyse der in demselben enthaltenen, zu den kleinen Gesichtsarterien tretenden Gefässzweige herbeigeführı habe.

Aus dem Mitgetheilten geht jedenfalls so viel hervor, dass die experimentellen Ergebnisse die Möglichkeit eines neurotischen Ursprungs der halbseitigen Gesichtshypertrophie zulassen. Die sichere Entscheidung darüber kann erst durch genauere Sectionen gebracht werden. In dem Friedreich'schen Fall konnte die Section leider nicht ganz eingehend gemacht werden, besonders fehlt auch jede mikroskopische Untersuchung, so dass das negative Resultat derselben nicht in Betracht gezogen werden kann.

Von klinischer Seite weisen die fast in allen Fällen beobachteten abnormen functionellen Symptome allerdings mit Sicherheit darauf hin, dass das Nervensystem in dieser oder jener Weise betheiligt sein muss. So kann der in drei von unseren fünf Fällen seit Geburt bestehende Speichelfluss nur auf abnorme Reizungszustände im Bereich der zugehörigen secretorischen Nervenapparate bezogen werden, seitdem durch die Physiologie nachgewiesen ist, dass die

3) Stilling, Untersuchungen über die Spinalirritation. 1840 . 
Speichelsecretion nur nach Reizung der secretorischen Nervenfasern eintritt, ohne eine solche aber völlig sistirt. Die einzige Ausnahme davou, die sog, paralytische Speichelabsonderung, kommt natürlich nicht in Betracht, weil dieselbe nach einiger Zeit unter Entartung der Drüse wieder nachlässt, wäbrend in unseren Fällen die abnorm vermehrte Speichelsecretion Jahre lang vorhanden war.

Gleiches gilt von der beobachteten übermässigen Secretion der Talgdrüsen, den neuralgischen Zahnschmerzen etc., was keiner weiteren Ausführung bedarf.

Es fehlt daher in der That der Trélat-Monod'schen Theorie von dem neurotischen Ursprung der Hemihypertrophie nicht an gewichtigen Stützen. Nur würde man gegenwärtig wohl weniger an die Gefässnerven wie Trélat-Monod denken als vielmehr an directe trophische Nerven. Ein abweichendes Verhalten des Gefässapparates kam überhaupt bisher nicht zur Beobachtung, wenn man davon absieht, dass in dem Falle von Friedreich die hypertrophische Wange öfters röther gefärbt war als die andere. Nur in dem Still ing'schen Fall machte sich ein abnormes Verhalten des Gefässapparates durch die zahlreichen dabei vorhandenen Gefässerweiterungen geltend und ist es hier wohl erlaubt, wie auch von Stilling gescheben, die Affection zu einem grossen Theil auf vasomotorische Einflüsse zurlickzufuhren, während in den ubrigen Fällen dies nicht zulässig erscheint. 